\title{
Adaptierbare Qualitätsbewertung bürgergenerierter Inhalte aus sozialen Medien
}

\author{
Christian Reuter, Michael Ritzkatis \\ Institut für Wirtschaftsinformatik, Universität Siegen
}

\section{Zusammenfassung}

Zwei Drittel aller Internetnutzer in Deutschland nutzen soziale Medien. Neben der Kommunikation mit eigenen Kontakten werden dort auch vermehrt Fotos, Filme und Inhalte ganz bewusst öffentlich geteilt. Vergangene Ereignisse, wie das Hochwasser in Mitteleuropa im Juni 2013, haben gezeigt, dass diese alltäglichen Verhaltensweisen ebenso in Ausnahmesituationen fortgeführt werden und bürgergenierte Inhalte auch dann beispielsweise über Facebook und Twitter verbreitet werden. Diese werden in Teilen von Behörden und Organisationen mit Sicherheitsaufgaben (BOS), wie der Polizei und Feuerwehr, ausgewertet und für die Lagebeurteilung genutzt. Dieser Beitrag stellt, basierend auf einer Analyse verwandter Arbeiten, die Ergebnisse einer empirischen Untersuchung zur Nutzung bürgergenerierter Inhalte und die Bedingungen für deren Integration in die Lagebeurteilung durch BOS dar. Die hieraus gewonnenen Erkenntnisse münden in Anforderungen an einen adaptierbaren Bewertungsmechanismus für bürgergenerierte Inhalte aus sozialen Medien.

\section{$1 \quad$ Einleitung}

Gemäß einer repräsentativen Studie des Bundesverbands Informationswirtschaft, Telekommunikation und neue Medien (BITKOM, 2013) sind mehr als drei Viertel der Internetnutzer in mindestens einem sozialen Netzwerk angemeldet, zwei Drittel sind aktive Nutzer. Auch wenn das Versenden privater Nachrichten die am häufigsten genutzte Funktionalität ist $(85 \%)$, kommen das Hochladen eigener Fotos und Filme (65\%) sowie das Teilen von Fotos, Videos, Texten oder Links $(65 \%)$ auf beachtliche Nutzungszahlen. Auch in Ausnahmesituationen, wie dem Love-Parade Unglück 2010 (Reuter et al., 2011), der Nuklearkatastrophe von Fukushima (Thomson et al., 2012) oder zuletzt dem mitteleuropäischen Hochwasser 2013 (Kaufhold \& Reuter, 2014), wurden soziale Medien von Betroffenen und Helfern genutzt, um Informationen bereitzustellen und auszutauschen. Doch (wie) werden diese Inhalte in der Gefahrenabwehr durch BOS genutzt? Und welche Anforderungen ergeben sich für die Gestaltung interaktiver Systeme zur Bewertung und Selektion dieser Inhalte? Basierend auf 
einer Darstellung relevanter Vorarbeiten (Kapitel 2) stellt dieser Beitrag die Ergebnisse einer empirischen Untersuchung zur Nutzung bürgergenerierter Inhalte durch BOS vor (Kapitel 3) und leitet Anforderungen für einen adaptierbaren Bewertungsmechanismus her (Kapitel 4).

\section{Lagebeurteilung mit sozialen Medien}

Informationen sind für die Beurteilung einer Schadenslage unerlässlich. Sie müssen zur richtigen Zeit, am richtigen Ort und in der richtigen Form vorhanden sein (Ley et al., 2012). Da Schadenslagen nicht nur zeitkritisch sondern auch einzigartig sind, bestehen ganz spezielle Informationsbedürfnisse, die im Vorfeld nicht bekannt sein können, und es fällt schwer, alle möglicherweise benötigten Informationen vorzuhalten (Turoff et al., 2004). Die Verfügbarkeit möglichst vieler Quellen, auf die ohne Verzögerung zugegriffen werden kann, erscheint notwendig. Gleichzeitig muss eine mögliche Informationsüberflutung verhindert werden, um die Entscheidungsfindung nicht zu beeinträchtigen (Hiltz \& Plotnick, 2013).

Neben automatisiert bereitgestellten Informationen (Wetterdaten, Pegelstände, etc.) können zwei verschiedene Klassen menschlicher Informationsquellen unterschieden werden: Einsatzkräfte vor Ort, welche angefragt werden können (Ludwig et al., 2013; Reuter \& Ritzkatis, 2013), sowie Personen oder Organisationen, die nicht aktiv an der Schadensbewältigung beteiligt sind. Bei einem Hausbrand kann die Anzahl der Bewohner über das Einwohnermeldeamt erfragt werden, wohingegen die Beurteilung der Größe des Feuers und möglicher Anwesender nur vor Ort begutachtet werden kann. Bei einem Stromausfall kann der Stromnetzbetreiber den Einsatzkräften schneller und besser Informationen über die betroffenen Gebiete zukommen lassen, als wenn diese vor Ort ermittelt werden würden (Ley et al., 2012). Die Bilder mancher Schäden finden sich jedoch in sozialen Medien. Durch diese Beispiele wird deutlich, dass extern beschaffte Informationen einerseits den Ablauf beschleunigen können und andererseits zum Teil vor Ort gar nicht ermittelt werden können. Nicht immer berichten Bürger dabei in Form von sachlichen Informationen. In einigen Fällen kommt es bei diesen subjektiven Informationsmeldungen dazu, dass eine gewisse Art von Selbstjustiz entsteht (Rizza et al., 2013). Daneben kann es zu Fehlinformationen kommen, wenn Situationen bewusst oder unbewusst fehlerhaft geschildert werden; sei es, weil sie einigen Aspekten nicht genügend Bedeutung widmen oder aber weil sie die Situation falsch wiedergeben (Thomson et al., 2012). Es gibt jedoch auch Informationen, die nicht auf alternative Weise beschafft werden können (Zagel, 2012). Zur Selektion und Nutzung bürgergenerierte Informationen existieren bereits einige Ansätze, die allerdings keine umfassende Qualitätsbewertung unterstützen:

- Twitcident (Terpstra et al., 2012) dient der Selektion von Tweets auf Basis von Schlagwörtern, der Art der Nachricht oder eines Nutzers mit anschließender Darstellung auf einer Karte. Derzeit bietet es leider keine Qualitätsbewertung anhand von Metainformationen wie bspw. dem Zeitpunkt der Erstellung - weiterhin bezieht es keine Informationen aus anderen sozialen Medien.

- SensePlace2 (Robinson et al., 2013) demonstriert ebenfalls eine Möglichkeit, georeferenzierte Informationen aus Tweets zu extrahieren und diese in einer Lagekarte 
darzustellen. Es handelt sich jedoch um eine umfängliche Erfassung ohne Qualitätsbewertung, die die Problematik der Informationsflut nicht adressiert.

- $\quad$ Tweet4act (Chowdhury et al. 2013) ermöglicht die Ermittlung und Klassifizierung von Informationen, die über Twitter verbreitet wurden. Dabei wird jede Nachricht mit einem schadenslagenspezifischen Wörterbuch in eine Schadensperiode klassifiziert. Hierfür wird sich spezieller Methoden des Maschinellen Lernens auf Basis von Wörterbüchern oder Sprachklassifizierungen.

- Mit TwitInfo (Marcus et al., 2011) können viele Informationen eines speziellen Ereignisses gesammelt, klassifiziert und visualisiert werden. Neben einer graphischen Visualisierung werden viele Informationen über die eigentliche Qualität der Informationen präsentiert. Eine persönliche Gewichtung der Qualitätsansprüche des Benutzers findet keine Anwendung.

- Mit Ushahidi (Mcclendon \& Robinson, 2012) können sich Bürger gegenseitig Informationen zukommen lassen. Darüber hinaus ist es prinzipiell möglich, die Daten auch Einsatzkräften zur Verfügung zu stellen. Die direkte Kommunikation und ungefilterte Informationsbereitstellung birgt jedoch die Gefahr einer Informationsflut und fordert den Empfänger zu einer manuellen Qualitätsbewertung auf.

- Mit Tweak the Tweet (Starbird \& Stamberger, 2010) wird die Auswertung und Klassifizierung der Informationen unterstützt. Obwohl diese Syntax verschiedene Qualitätsbewertungen ermöglicht, verhindert die Auswertung von Informationen nur eines bestimmten Formats auch eine übersichtliche Lagedarstellung.

Zusammengefasst lässt sich feststellen, dass bereits viele Studien und Ansätze den Umgang mit bürgergenerierten Inhalten thematisieren, jedoch die Thematik der Qualitätsbewertung, wenn überhaupt, nur rudimentär behandeln. Um die tatsächliche Nutzung bürgergenerierter Inhalte und die Bedingungen für deren Integration in die Lagebeurteilung von BOS zu erarbeiten, erscheint eine empirische Studie sinnvoll.

\section{Empirische Studie: Nutzung bürgergenerierter Inhalte}

Die dieser Arbeit zugrunde liegende empirische Studie der Informations- und Kommunikationsaktivitäten von BOS (hier insbesondere der Feuerwehr und Polizei) sowie Infrastrukturbetreiber (Stromnetz) wurde 2010 bis 2012 in zwei geographisch und strukturell verschiedenen Kreisen Nordrhein-Westfalens durchgeführt. Es wurden verschiedene qualitative Methoden wie Dokumentenanalysen, Beobachtungen, teilstrukturierte Interviews und Gruppendiskussionen durchgeführt und ausgewertet. Neben einer Fokussierung auf Improvisationspraktiken (Ley et al., 2012), der Zusammenarbeit örtlich verteilter Kräfte (Reuter \& Ritzkatis, 2013) und der Reporting-Praktiken (Ludwig et al., 2013) wurde die Kooperation mit Bürgern und die Nutzung bürgergenerierter Inhalte thematisiert.

Ziel der Dokumentenanalyse war die Untersuchung offizieller Arbeitsabläufe mit Hilfe von Gesetzen und Richtlinien. Die Beobachtungen dienten dazu, Erkenntnisse über die tatsächliche praktische Arbeit zu erhalten. Diese wurden in der Leitstelle während eines normalen Arbeitstages (neun Stunden), im Krisenstab und der Einsatzleitung einer Krisenübung (vier Stunden) und eines Großereignisses (sechs Stunden) durchgeführt. Die 22 semi- 
strukturierten Interviews (je 90 Minuten; nummeriert als I01-22) dienten der Reflexion der Arbeitspraxis, der Identifikation möglicher IT-Unterstützung und interorganisationaler Informationsbedarfe und wurden mit Einsatzkräften verschiedener Ebenen durchgeführt. Sie fokussierten im ersten Teil die Arbeitstätigkeiten in normalen Lagen, im zweiten Teil potentielle Aufgaben im vorgelegten Szenario und im dritten Teil genutzte Informations- und Kommunikationstechnologien. Fünf ergänzende Interviews (je 60 Minuten; nummeriert als IM01-05) fokussierten mobile Kollaborationspraktiken. Die vier Gruppendiskussionen (je vier Stunden, nummeriert als W1-4) dienten der Erfassung kommunikativer Arbeitspraxen. Bei der Auswertung des vorliegenden Datenmaterials wurde sich an der Grounded Theory (Strauss, 2004) orientiert. Dazu wurden die Transskripte offen kodiert und die Aussagen in Textbausteine und spätere Kategorien aufgeteilt.

\subsection{Nutzung sozialer Medien zur Lagebeurteilung}

Generell kann eine Lagebeurteilung nicht nur auf Basis der organisationsinternen Informationen stattfinden. Externe Informationen können einen Informationsgewinn darstellen (W3). Insbesondere werden neben textuellen Informationen gerne von Bürgern veröffentlichte Bilder verwendet, mit denen der Schadenshergang bzw. der aktuelle Zustand besser beurteilt werden kann: „, Twitter ist ja häufig schneller mit Informationen als das bei uns über Polizeifunk oder Handy gelaufen ist. Wenn ich das bei Demogeschehen oder sonstiges sehe. [...] Auch diese sind häufig mit Bildern oder Videos hinterlegt im Internet zu finden bei aktuellen Ereignissen. Also da werden wir in Zukunft deutlich mehr mit zu tun haben, da bin ich mir ganz sicher" (I2). Ein Beispiel ist die Nachbetrachtung eines Ereignisses: „, Unsere Ermittler, die nutzen diese Brandbilder ganz gerne, weil unsere Kripo ist natürlich in den Entstehungsphasen eines Brandes oder so sind die ja nicht vor Ort. Die sind natürlich dann sehr oft angewiesen [auf diese] [...] um diesen Brandverlauf dann noch einmal zu sehen " (I02).

Informationen müssen aber nicht immer hilfreich sein: „Informationen bringen immer dann was, wenn sie mein Verhalten beeinflussen. Immer dann, wenn Informationen da sind, die keinen Einfluss auf mein Verhalten haben, ist es eine Reizüberflutung “ (I06). Daher wird versucht, vor allem relevante Informationen zu sammeln: „, Wir versuchen ja von jedem Anrufer Informationen zu bekommen “(I15). Auch im Einsatzgeschehen selbst werden Personen vor Ort aktiv und unterstützen die Einsatzkräfte: „Es sind viele spezielle Sachen, wo man Grundkenntnisse oder Vorwissen haben sollte, aber es gibt auch viele Sachen, wo man auf Wissen und Fähigkeiten der Bürger zurückgreifen kann"(I11). Abgesehen davon haben die Einsatzkräfte auch ganz besondere Anforderungen an alle Informationen, denn ,eine Information in einer Einsatzleitung z. B. zu haben, heißt nicht, dass man mit der Information auch irgendetwas machen kann" (I06). Ungeachtet der Informationsmasse übt der Zeitfaktor erheblichen Druck auf die Einsatzkräfte aus. Daher ist es immens wichtig, dass jegliche Operation zeitnah durchgeführt wird. Daher besteht ,keine Zeit mich mit Fremden noch zu beschäftigen " (I02). Die Fehlerhaftigkeit bürgergenerierter Informationen bleibt jedoch bestehen und macht deshalb eine Bewertung notwendig. 


\subsection{Selektion, Verarbeitung und Qualitätsbewertung}

Im Einsatzgeschehen stellt sich die Frage: ,, Wer bewertet das nun [...] und bringt uns das überhaupt in der Lagebeurteilung vorwärts? " (I03). Vor allem die Masse der bürgergenierten Inhalte macht deren Nutzung problematisch: „Und vor allen Dingen ist von den 300 ja 290 Müll. Da kannste aus zehn Informationen was für die raus ziehen“ (I02). Bei der Masse entsteht schnell das Problem, diese geeignet zu verarbeiten „Du musst die halt alle lesen. Wenn da eine Vorgliederung da wäre, wäre das natürlich sehr hilfreich“ (I02). Aus diesem Grund ist eine automatische Filterung empfehlenswert: „Es wäre schön, wenn man einen Filter drin hätte, der die wichtigen von den unwichtigen trennt" (I03). Jedoch ist eine gewisse quantitative Häufigkeit einer Information notwendig, damit diese von den Einsatzkräften als glaubwürdig erachtet werden kann: „Wenn ich eine Quelle habe, ist das ein Problem. Wenn ich fünf Quellen habe, ist das sicherlich seriöser als eine“ (I15). Insbesondere sind externe Quellen für Fehlinformationen anfällig (I14, I15) und müssen deshalb überprüft werden (I15): Man ,,muss vorsichtig sein mit dem was drin steht, weil es nicht immer so ist, wie es wirklich ist“ (I14) - „Da zeigt sich natürlich immer wieder, dass wir da aufs Glatteis geführt werden sollen [...] und wir müssen die Informationen bewerten für uns" (I02). Dabei handelt es sich nicht immer um bewusste Fehlinformationen, sondern um die Art der subjektiven Wahrnehmung einer Situation, die für einen neutralen Betrachter ein anderes Bild ergeben kann. Insgesamt scheitert der Einsatz von Bürgerinformationen aus sozialen Medien an der Bewertung der Informationen durch Einsatzkräfte, denn ,,da ist einfach irgend so ein Flaschenhals, über den wir nicht drüber kommen “ (I02).

Insgesamt ist erkennbar, dass ,je präziser die Informationen sind, desto besser sind die [Informationen] “ (I02). Diese Präzision der Informationen kann durch eine Bewertung sichergestellt werden. Insbesondere muss sichergestellt sein, dass die gefilterten Informationen einen Mehrwert für die Einsatzkräfte darstellen (I02, I03). Eine globale Filterung erweist sich auch als schwierig, ,weil was für den Abschnittsführer wichtig ist, das kann man, glaube ich, im Vorfeld nicht unbedingt filtern. Das kann sein, dass er die gleichen Informationen benötigt wie die Einsatzleitung" (IM01). Daher muss eine Flexibilität der Bewertungskriterien gegeben sein (I19). Die persönliche Auswahl der Informationen muss aufgrund des zeitkritischen Aspekts von Schadenslagen unterstützt werden, denn jede Einsatzkraft muss „relativ schnell das Wichtige vom Unwichtigen“ (I19) trennen.

Das erstellte Bild muss auf jeden Fall eine gewisse Aussagekraft widerspiegeln und hilfreich bei der Beurteilung der Situation sein: ,Wenn der jetzt ein Foto von einem Fenster macht, weiß ich, der hat da gestanden. Aber wo befindet sich das Fenster?" (I16). Also benötigen die Bilder genau wie normale Textinformationen weitere Metainformationen. Bilder können gerade im Szenario der Beurteilung von Menschenmassen bei Großveranstaltungen sehr hilfreich sein: „Hätte man festgestellt, dass sich an bestimmten Bereichen ein Personenstau ergeben hätte, hätte im oberen Bereich, also im Zulauf, sofort durch die Security der Zulauf gesperrt werden können " (I06). Obwohl sich daraus motiviert, dass viele Informationen gesammelt werden sollten, wissen ,,die meisten Leute [...] ja gar nicht, worauf es ankommt und was für Informationen wir haben müssten " (I02). Somit besteht die Gefahr, dass Informationen keinen Mehrwert bieten und auch im Einsatzgeschehen nicht genutzt werden können: „Ich glaube kaum, dass jemand, der nicht aus dem polizeilichen oder aus dem Feuer- 
wehrbereich kommt, in der Lage ist, in so einer Stresssituation notwendige Informationen zu liefern “ (I02). Ein unausgebildeter Bürger kann dieses Wissen in den seltensten Fällen besitzen. „Von daher muss man mit solchen Informationen sehr vorsichtig umgehen“ (I14).

Letztlich muss die Einsatzkraft die Verantwortung über die Handlungen übernehmen und entscheiden, ob eine Information verwendet wird oder nicht (I15). Fehlbewertungen sind sowohl durch Menschenhand als auch durch Computerunterstützung möglich. Egal wie gut durchdacht der Bewertungsmechanismus ist, es wird ,ein Restrisiko [geben] und das muss der Verantwortliche tragen, so einfach ist das " (I15). Aus diesem Grund sind die Einsatzkräfte auch sehr vorsichtig in der Benutzung von externen Informationen. Abschließend lässt sich festhalten, dass „Informationen zu bewerten, richtig zu bewerten und damit umzugehen [...] eine sehr anspruchsvolle Aufgabe ist " (I15). Jede einzelne Information ist dabei wie ein Baustein zur Erstellung des gesamten Lagebildes zu betrachten: „Also immer mehr an dieses Skelett, was man zunächst hat, Fleisch dran zu bringen, damit man nachher ein Bild hat, nicht nur eine Silhouette, sondern eine Gestalt und dass diese Gestalt dann maßgeblich ist für irgendwelche polizeiliche Maßnahmen " (I16). Die Lagebeurteilung beeinflusst also die Maßnahmen, die die Lage wiederum verändern können. Jedoch ist eine Erhöhung der Masse an Informationen nicht sinnvoll, denn Informationen sind nicht immer brauchbar.

\section{Adaptierbare Bewertung bürgergenerierter Inhalte}

\subsection{Synopse der Anforderungen}

Die Literatur sowie die empirische Studie haben gezeigt, dass die Bewertung der Masseninformationen und die Extraktion der relevanten Informationen die größte Herausforderung darstellen. Auf dieser Basis wurde ein Bewertungsmechanismus für soziale Medien erstellt.

- Plattformübergreifende Suche: Durch eine offene Konzeptionierung können prinzipiell verschiedene Quellen für die Informationssuche einbezogen werden. So ergibt sich eine große Zeitersparnis, da die verschiedenen Quellen nicht separat durchsucht werden müssen. Die ebenfalls daraus resultierende größere Bandbreite kann eine bessere Verifizierung der vorhandenen Informationen auf Richtigkeit ermöglichen. So können einerseits verschiedene Informationstypen sowie andererseits ein umfassenderes Lagebild entstehen.

- Automatisierte Verarbeitung: Die Masse von bürgergenerierten Informationen macht eine manuelle Verarbeitung in zeitkritischen Schadenslagen schwierig. Daher werden die Informationen automatisiert nach den Vorgaben des Benutzers verarbeitet. Im Idealfall muss er nur wenige und insbesondere für ihn relevante Informationen betrachten.

- Flexible und adaptierbare Bewertung: Unsere empirische Studie hat gezeigt, dass unterschiedliche Schadenslagen unterschiedliche Informationen in verschiedenen Verdichtungen erfordern. Zusätzlich sind Qualitätsanforderungen immer abhängig vom Nutzer. Daher wird die Wahl und Gewichtung der Bewertungsmethoden allein in das Ermessen des Benutzers gelegt. 
- Nachvollziehbarkeit der Ergebnisse: Um das Vertrauen der Benutzer in den Automatismus der Qualitätsbewertung zu erhöhen, wird dieser Vorgang so transparent wie möglich gestaltet. Es soll für den Benutzer nachvollziehbar sein, warum eine Information wie bewertet wurde. Dadurch begibt er sich nicht in eine Abhängigkeit eines geschlossenen Bewertungssystems, denn letztendlich muss die Einsatzkraft die Verwendung der Information verantworten.

\subsection{Realisierung eines adaptierbaren Bewertungsservices}

Zur Realisierung eines adaptierbaren Bewertungsservices wurden verschiedene Technologien verwendet. Spezielle APIs dienen dem Zugriff auf die Inhalte aus sozialen Medien derzeit die Twitter Streaming API (https://dev.twitter.com/docs/api/streaming), Twitter REST API (https://dev.twitter.com/docs/api) sowie Facebook Graph API (https://developers. facebook.com/docs/graph-api). Die Inhalte werden anschließend in das OpenSocial Format (http://opensocial.org/) konvertiert. Das eigentliche Bewertungstool ist mit einer serviceorientierten Architektur (SOA) unter Zuhilfenahme des Apache Tomcat, zur Zentralisierung der Bewertung, realisiert. Eine Bereitstellung der bewerteten Daten in einem allgemeinem Format (hier: JavaScript Object Notation - JSON) ermöglicht eine Einbindung in verschiedene Architekturen.

Alle übermittelten Inhalte werden hinsichtlich jedes selektierten Bewertungskriteriums mit einem Maß des relativen Erfüllungsgrades, gemessen an der schlechtesten und besten Information in der Datensammlung, bewertet. Diese Bewertung kann sowohl auf Basis von Metadaten als auch mit Hilfe von Text-Mining-Methoden stattfinden. Insgesamt stellt das Bewertungstool 15 Bewertungsmethoden bereit, die sich in folgende vier Kategorien unterteilen lassen:

1. Metadaten: Autorenhäufigkeit, zeitliche Nähe, örtliche Nähe, Anzahl Follower/Likes, Metadaten

2. Inhalt (syntaktischer und semantischer Aufbau der Information sowie das Vorkommen gewisser Wortsequenzen): Suchbegriffhäufigkeit, Stopwords

3. Klassifikation (semantische Bedeutung): Sentiment Analyse, Fear Factor, Happiness Factor

4. Wissenschaftliche Methoden (statistische Verfahren): Shannonscher Informationsgehalt, Term Frequency - Inverse Document Frequency

Von diesen Bewertungsmethoden kann die Anwendung wählen, welche sie mit welchem Gewicht verwenden möchte. Durch diese Auswahl und die verschiedenen Kategorien wird ein Einsatz des Bewertungstools in einer Vielzahl von unterschiedlichen Einsatzszenarien möglich. Die Ergebnisse inklusive der Originaldaten werden in JSON bereitgestellt und können weiterverarbeitet werden.

\subsection{Prototypische Integration in eine Facebook-Applikation}

Zur Illustration der Funktionalität wurde dieses prototypisch in eine Facebook-Anwendung, die ungebundene Helfer in ihrer Arbeit der Schadensbewältigung durch die medienübergreifende Informationssuche und die Informationsgenerierung unterstützen soll, integriert 
(Kaufhold \& Reuter, 2014). Innerhalb dieser Anwendung werden dem Benutzer die 15 Bewertungsmethoden durch vereinfachte sprachliche Beschreibungen statt durch formale Definitionen erläutert. Bei jedem gibt der Benutzer die persönliche Wichtigkeit über einen stufenweise einstellbaren Schieberegler an (Abbildung 1). Dazu werden bereitgestellte Schnittstellen des Bewertungsservices verwendet, sodass eine zentrale, serverseitige Qualitätsbewertung möglich ist.

\section{SUCHEINSTELLUNGEN}

\begin{tabular}{|ll|}
\hline Allgemein & \\
Suchbegriff: & Hochwasser Elbe \\
Netzwerke festlegen: & $\square$ Facebook $\square$ Twitter $\square$ Evaluation \\
Zeitraum angeben: & 2013-12-05 00:00-2013-12-07 00:00 \\
Standort auswählen: $\mathbf{B}$ & Lauenburg Elbe. Deuts \\
Umkreis der Suche $(\mathrm{km}):$ & 20.0
\end{tabular}

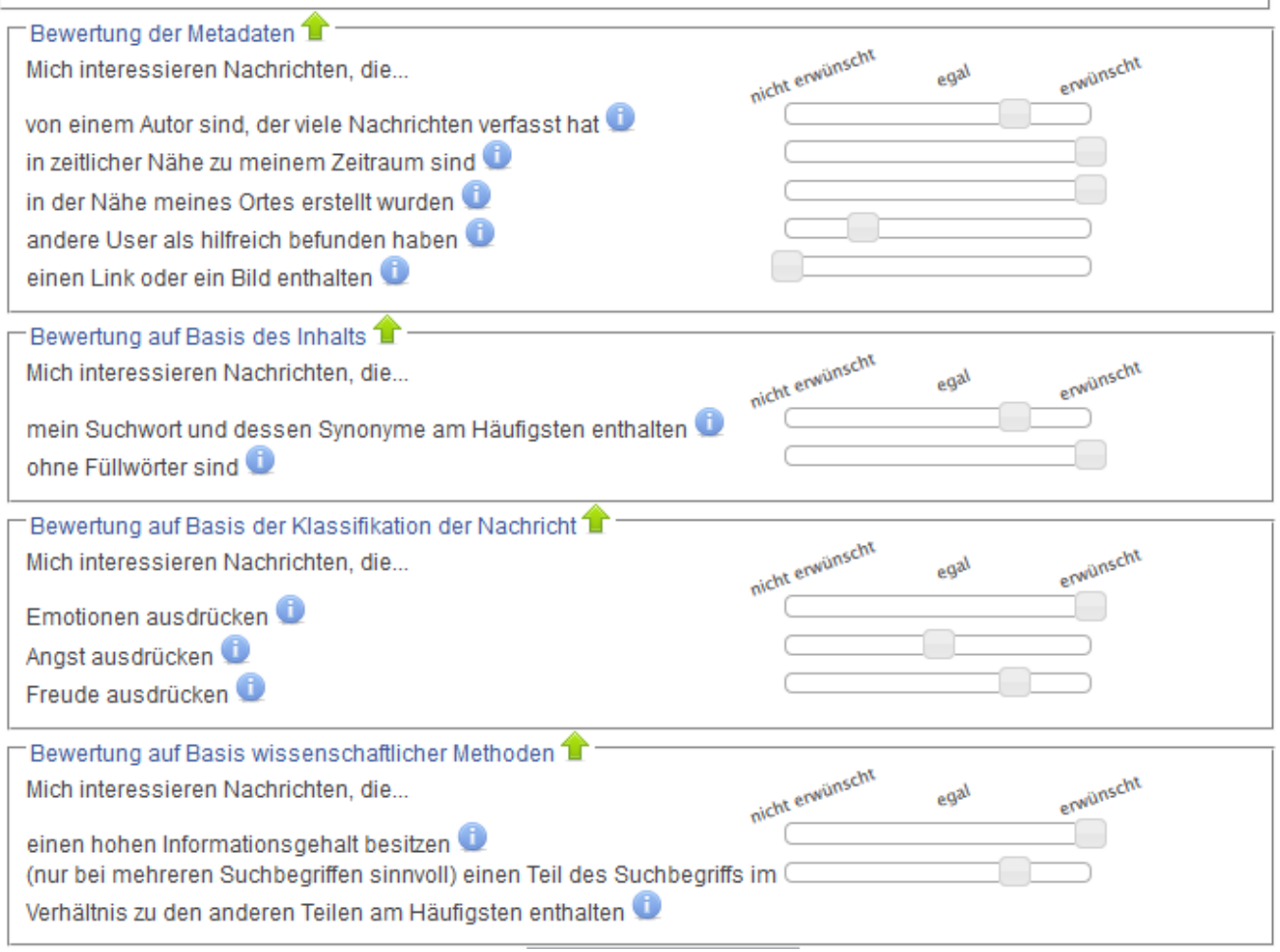

Abbildung 1: Adaptierbare Informationssuche und Qualitätsbewertung mithilfe von Schiebereglern

Die Ergebnisse dieser Informationssuche und Bewertung werden sowohl tabellarisch mit verschiedenen Metainformationen, die als MouseOver-Text angezeigt werden, als auch visuell auf einer Lagekarte zur direkten Bestimmung der Nähe der Information zum Suchstandort dargestellt. Bei jeder Information wird die Bewertung jedes einzelnen Bewertungskriteriums dem Benutzer in Form eines Ampelsystems zur Verfügung gestellt. (Abbildung 2). 


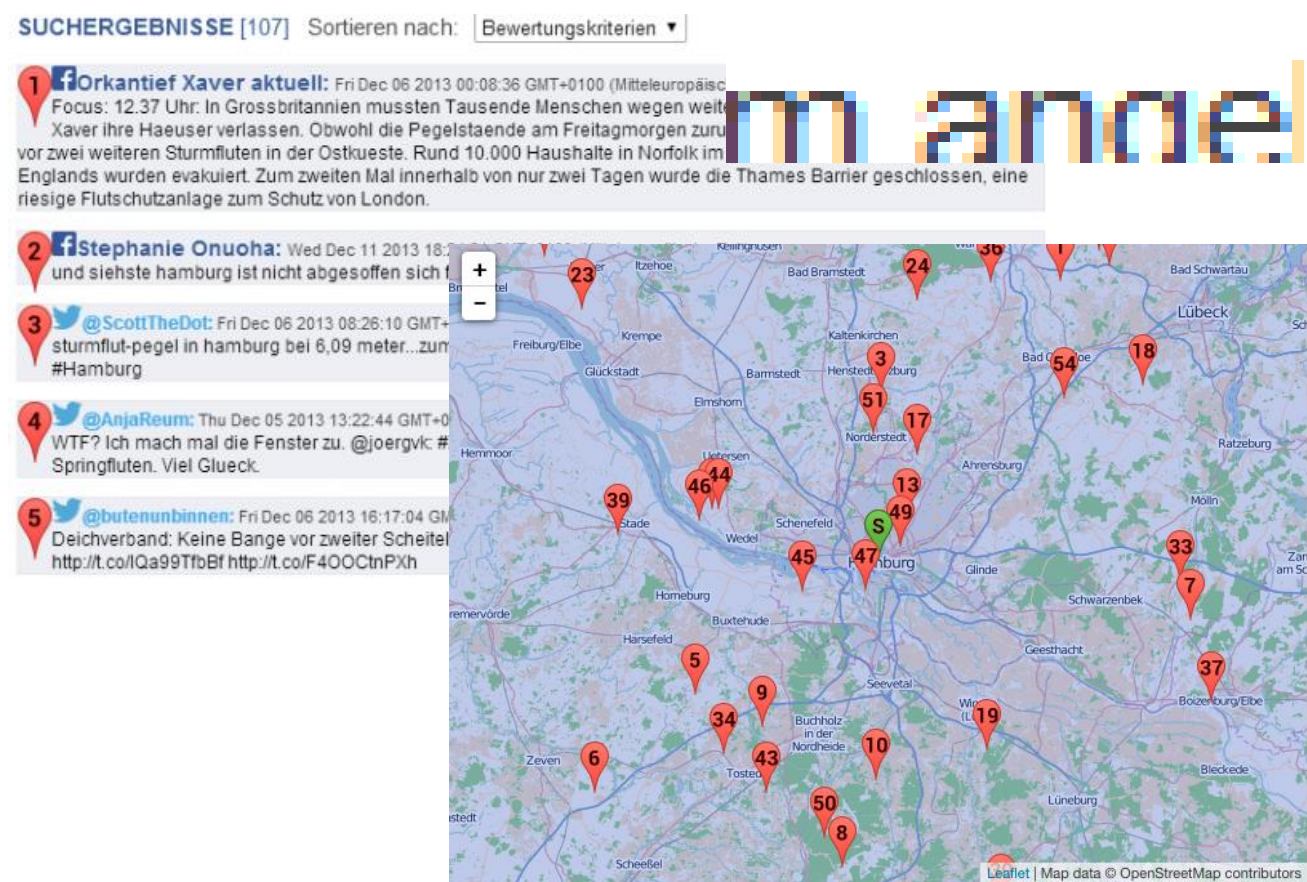

Abbildung 2: Suchergebnisse (links) inkl. Ampelsystem (oben rechts) und Darstellung auf der Karte (rechts)

\section{$5 \quad$ Fazit}

Dieser Artikel betrachtet die adaptierbare Qualitätsbewertung bürgergenerierter Inhalte aus sozialen Medien. Der Kernbeitrag stellt eine empirische Studie zum Umgang mit bürgergenerierten Inhalten in Schadenslagen dar. Hierdurch wurden die derzeitigen Hindernisse sowie Anforderungen an eine adaptierbare Qualitätsbewertung deutlich. Diese münden in ein Konzept eines adaptierbaren Bewertungsservices, der flexibel in verschiedene Anwendungen integriert werden kann. Gegenüber den im Beitrag vorgestellten Ansätzen (Twitcident, SensePlace2, Tweet4act, TwitInfo) werden nicht nur Daten aus genau einem Netzwerk, insbesondere Twitter, verwendet, sondern unterschiedliche Informationsquellen unterstützt. Im Unterschied $\mathrm{zu}$ Tweak the Tweet werden aus den Informationsquellen sämtliche Informationen und nicht nur eines gewissen Formats betrachtet. Eine Evaluation, die nicht Bestandteil dieses Beitrags ist, soll den praktischen Nutzen adaptierbarer Qualitätsbewertung ergründen und die Möglichkeiten der Artikulation von Qualitätsanforderungen erforschen.

Gefördert im Rahmen des EU-FP7-Projekts „EmerGent“ (Fördernummer 608352).

\section{Literatur}

BITKOM. (2013). Soziale Netzwerke 2013 - Eine repräsentative Untersuchung zur Nutzung sozialer Netzwerke im Internet. Berlin, Germany. 
Chowdhury, S., Amer-Yahia, S., Castillo, C., Imran, M., \& Asghar, M. R. (2013). Tweet4act: Using Incident-Specific Profiles for Classifying Crisis-Related Messages. In Proc. ISCRAM (pp. 834839). Baden-Baden, Germany.

Hiltz, S., \& Plotnick, L. (2013). Dealing with Information Overload When Using Social Media for Emergency Management: Emerging Solutions. In Proc. ISCRAM (pp. 823-827). Baden-Baden.

Kaufhold, M.-A., \& Reuter, C. (2014). Vernetzte Selbsthilfe in Sozialen Medien beim Hochwasser 2013 in Deutschland. I-Com - Zeitschrift Für Interaktive und Kooperative Medien, 13(1), 20-28.

Ley, B., Pipek, V., Reuter, C., \& Wiedenhoefer, T. (2012). Supporting Improvisation Work in InterOrganizational Crisis Management. In Proc. CHI (pp. 1529-1538). Austin, USA: ACM Press.

Ludwig, T., Reuter, C., \& Pipek, V. (2013). What You See Is What I Need: Mobile Reporting Practices in Emergencies. In Proc. ECSCW (pp. 181-206). Paphos, Cyrus: Springer.

Marcus, A., Bernstein, M., \& Badar, O. (2011). Twitinfo: aggregating and visualizing microblogs for event exploration. Proc. CHI, Vancouver, Canada.

Marino, T., Nascimento, B. do, \& Borges, M. (2012). GIS Supporting Data Gathering and Fast Decision Making in Emergencies Situations. Proc. ISCRAM - Vancouver, Canada, 1-6.

McClendon, S., \& Robinson, A. C. (2012). Leveraging Geospatially-Oriented Social Media Communications in Disaster Response. In Proc. ISCRAM. Vancouver, Canada.

Reuter, C., Marx, A., \& Pipek, V. (2011). Desaster 2.0: Einbeziehung von Bürgern in das Krisenmanagement. In Mensch \& Computer 2011 (pp. 141-150). München: Oldenbourg-Verlag.

Reuter, C., \& Ritzkatis, M. (2013). Unterstützung mobiler Geo-Kollaboration zur Lageeinschätzung von Feuerwehr und Polizei. In Proc. WI (pp. 1877-1891). Leipzig, Germany.

Rizza, C., Pereira, Â., \& Curvelo, P. (2013). Do-it-yourself Justice-Considerations of Social Media use in a Crisis Situation: The Case of the 2011 Vancouver Riots. Proc. ISCRAM, Baden-Baden, Germany, 411-415.

Robinson, A., Savelyev, A., Pezanowski, S., \& MacEachren, A. M. (2013). Understanding the Utility of Geospatial Information in Social Media. Proc. ISCRAM. Baden-Baden, Germany, 918-922.

Starbird, K., \& Stamberger, J. (2010). Tweak the Tweet: Leveraging Microblogging Proliferation with a Prescriptive Syntax to Support Citizen Reporting. In Proc. ISCRAM. Seattle, USA.

Strauss, A. L. (2004). Methodologische Grundlagen der Grounded Theory. In J. Strübing \& B. Schnettler (Eds.), Methodologie interpretativer Sozialforschung: Klassische Grundlagentexte (pp. 427-453). Konstanz, Germany: UVK.

Terpstra, T., Vries, A. de, Stronkman, R., \& Paradies, G. L. (2012). Towards a realtime Twitter analysis during crises for operational crisis management. In Proc. ISCRAM. Vancouver, Canada.

Thomson, R., Ito, N., Suda, H., \& Lin, F. (2012). Trusting Tweets: The Fukushima Disaster and Information Source Credibility on Twitter. In Proc. ISCRAM (pp. 1-10). Vancouver, Canada.

Turoff, M., Chumer, M., van de Walle, B., \& Yao, X. (2004). The design of a dynamic emergency response management information system (DERMIS). JITTA, 5(4), 1-35.

Zagel, B. (2012). Soziale Netzwerke als Impulsgeber für das Verkehrs-und Sicherheitsmanagement bei Großveranstaltungen. In A. Koch, T. Kutzner, \& T. Eder (Eds.), Geoinformationssysteme (pp. 223 232). Berlin/Offenbach: VDE Verlag. 